\title{
SCIDAR: an optical turbulence profiler for Dome A
}

\author{
Li-Yong Liu ${ }^{1,2}$, Yong-Qiang Yao ${ }^{1}$, Jean Vernin ${ }^{2}$, Merieme Chadid ${ }^{2}$, \\ Hong-Shuai Wang ${ }^{1}$ and Yi-Ping Wang ${ }^{1}$ \\ ${ }^{1}$ National Astronomical Observatories, Chinese Academy of Sciences, \\ 20A Datun Road, Chaoyang District, Beijing, China \\ email: liuly@nao.cas.cn \\ ${ }^{2}$ Université de Nice-Sophia Antipolis, Observatoire de la Côte d'Azur, CNRS-UMR7293, Lab. \\ Lagrange, \\ Parc Valrose, 06108, Nice Cedex 2, France \\ email: jean.vernin@unice.fr
}

\begin{abstract}
This paper introduces a plan to detect turbulence profiles at Dome A with a Single Star Scidar (SSS), to enhance our understanding of the characteristics of the site. The development of a portable monitor for profiling vertical atmospheric optical turbulence and wind speed is presented. By analyzing the spatial auto and cross-correlation functions of very short exposure images of single star scintillation patterns, the SSS can provide the vertical profiles of turbulence intensity $C_{n}^{2}(h)$ and wind speed $V(h)$. A SSS prototype is already operational at Ali in Tibet which will be improved in order to become fully robotic and adapted to extreme weather conditions that prevail at Dome A in Antarctica.
\end{abstract}

Keywords. Scidar, Optical turbulence, Dome A

\section{Introduction}

On the Antarctic plateau, Dome A benefits from exceptional atmospheric conditions, such as good seeing, coldness and very low precipitable water vapour, which potentially makes it one of the best sites for an astronomical ground-based observatory. A Chinese team first landed on Dome A, in January 2005, and returned again to Dome A in 2008 (Yang et al.2009). The so-called "PLATO" observatory has been installed in order to carry out a site testing campaign, which comprises a set of site testing instruments to characterize the Dome A site (Lawrence et al. 2008).

Two instruments have been set up to quantify the optical turbulence at Dome A. One is a Surface layer NOn-Doppler Acoustic Radar (SNODAR) to measure the turbulence within the near-surface layer (Bonner et al. 2010), the other is a Differential Image Motion Monitor (DIMM) to evaluate the seeing quality (Pei et al. 2010). We propose to develop a Single Star Scidar (SSS) in order to achieve a more detailed characterization of the optical turbulence within the boundary layer as well as the free atmosphere at Dome A. The SSS is a portable monitor, which can retrieve profiles of optical turbulence from the ground to the top of atmosphere. It has been running for more than one year at Dome C, as reported by Vernin et al. 2009 and Giordano et al. 2012.

\section{Plan to install a SSS}

The National Astronomical Observatories (NAOC) and the University of Nice Sophia Antipolis (UNSA) have been working together to develop a new SSS instrument for Dome A since 2009. We carried out a series of SSS experiments under real-sky conditions. We 


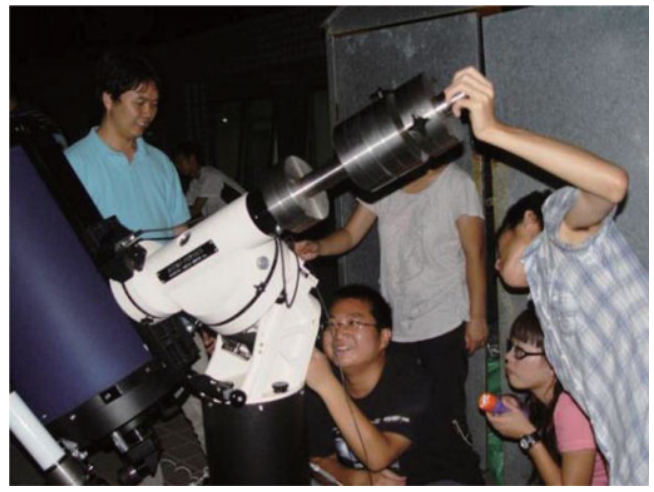

Figure 1. First SSS experiment at NAOC during summer 2010.

performed the first experiment with the new SSS at the headquarters of NAOC (Fig.1), and turbulence measurements with the SSS have been successfully carried out at Xinglong Observatory in China. The same SSS has been moved to Ali observatory, Tibet, at an altitude of $5,100 \mathrm{~m}$, and for the first time, we retrieved the turbulence and wind profiles above the Tibetan plateau in November, 2011 (Liu et al.2012). We plan to install the SSS to Dome A after "antarcticization" of this instrument.

\section{Improvement for Dome A}

Due to the harsh environmental conditions in Antarctica, the SSS instrument robotization is important in order to guarantee continuous measurements during the Antarctic winter. An automatic guider has been developed to track the target star for a long time, as detailed in Liu et al. 2012. We are also committed to the development of remote control technology, which can monitor and diagnostics the SSS instrument via the Internet, and automatic download of the measurements. Cold temperatures, as low as $-80^{\circ} \mathrm{C}$, might be fatal for the instrument, and an antarcticization procedure is foreseen to ensure that the telescope mount and CCD camera will work well even at low temperatures.

\section{Conclusions}

We have developed a new SSS in order to characterize the optical turbulence at Dome A. The instrument was successfully tested under real-sky conditions in various observatories. We are committed to developing a fully robotic version of the SSS, which will be suitable for turbulence measurements even under extreme environment that prevail at Dome A.

Acknowledgements: This work is supported by the National Natural Science Foundation of China (NSFC, Grant Nos. 10903014 and 11073031).

\section{References}

Yang, H., Allen, G., Ashley, et al., 2009, PASP, 121, 174-184.

J. S. Lawrence, G. R. Allen, M. C. B., Ashley, et al., 2008, Proc. SPIE, 7012, 701227-701227-12.

C. S. Bonner, M. C. B., Ashley, X. Cui, et al., 2010, PASP, 122, 1122-1131.

C. Pei, H. Chen, X. Yuan, et al., 2010, Proc. SPIE, 7733, pp. 77334W-77334W-8.

Vernin, J., Chadid, M., Aristidi, E., et al., 2009, A\&A, 1271, 1276

Giordano, C., Vernin, J., Chadid, M., et al., 2012, PASP, 494, 506

L. Liu, Y. Yao, J. Vernin, et al., 2012, Proc. SPIE, 8444, pp. 844464 (1-7) 